\title{
René Metz
}

\section{Les responsables des biens des Églises dans la perspective de Vatican II comparée a celle du Code de 1917}

Prawo Kanoniczne : kwartalnik prawno-historyczny 20/1-2, 53-65

1977

Artykuł został zdigitalizowany i opracowany do udostępnienia w internecie przez Muzeum Historii Polski w ramach prac podejmowanych na rzecz zapewnienia otwartego, powszechnego i trwałego dostępu do polskiego dorobku naukowego i kulturalnego. Artykuł jest umieszczony w kolekcji cyfrowej bazhum.muzhp.pl, gromadzącej zawartość polskich czasopism humanistycznych i społecznych.

Tekst jest udostępniony do wykorzystania w ramach dozwolonego użytku. 
Prawo Kanoniczne

20 (1977) $\mathrm{nr} 1-2$

RENE METZ

UNIVERSITE DE STRASBOURG

\section{LES RESPONSABLES DES BIENS DES EGLISES DANS LA PERSPECTIVE DE VATICAN II COMPAREE A CELLE DU CODE DE 1917}

\section{Les motifis du changement d'orientation}

Quelques remarques préliminaires s'imposent pour bien préciser le sujet de la présente étude. Dans le titre, il est question des personnes qui ont la respon'sabilité des biens des églises. Or, la fonction la plus importante qui incombe aux responsables des biens est ladministration de ces biens au mieux des intérêts du propriétaire. La question qui nous intéresse donc en l'occurrence est de connaitre les personnes qui sont chargées d'administrer les biens appartenant aux églises et qui, par conséquent, sont habilitées à conserver ces biens, à les gérer, à les faire fructifier, à décider de leur utilisation pour les divers besoins auxquels ils sont destinés.

En outre, le titre mentionne les biens des églises sans autre précision. En fait, nous nous en tiendrons aux églises paroissiales; l'exposé sera plus concret et le sujet, moins vaste. Mais ce que nous dirons des biens églises paroissiales vaut, mutatis mutandis, pour les biens des autres églises, par exemple les églises diocésaines. Les paroisses, auxquelles nous nous intéressons plus directement, jouissent en droit canonique de la personnalité jurdique, comme l'indique le canon $1495 \S 2$ du Code de 1917. Elles sont donc aptes, juridiquement, à posseder des biens. L'origine de ceś biens est multiple; dons des fidèles, produits de quêtes et de troncs, rentes, subventions d'organismes publics ou privés. La destination et l'utilisation de ces fonds sont, elles aussi, très variées: entretien des bâtiments, frais du culte et du personnel, chauffage, éclairage, oeuvres de charité.

Tel est le cadre précis de cette étude: les églises paroissiales et les personnes que le droit canonique habilite à gérer, effectivement, les biens appartenant à ces églises. Or, sur ce point, nous constatons une différence très nette entre la législation de 1917 et les directives données par le Ile concile du Vatican. Nous n'avons pas l'intention de décrire le mécanisme de cette administration tel que le prévoit le Code de droit canonique et que le suggére Vatican II. Nous voudrions élever le débat et dégager les principes qui sont 
à l'origine des dispositions prises par le législateur de 1917 et les nouvelles orientations qui se dessinent dans les textes du concile. Il nous importe de connaître les idées qi sont sous-jacentes aux deux conceptions: celle du Code de 1917 et celle du Ile concile du Vatican.

A cette fin, nous exposerons tout d'abord la maniére dont de Code de droit canonique concevait la responsabilité de cette administration pour la comparer à la manière dont la voit le IIe concile du Vatican. La comparaison des deux conceptions laisse apparaître un changement complet d'orientation. Ensuite, nous nous efforcerons de rechercher précisément les raisons qui ont motivé cette double manière d'envisager la même institution.

\section{Les dispositions du Code de 1917 et les nouvelles orientations données par Vatican II}

Le Codex iuris canonici adopte une position très claire au sujet de la responsabilite concernant la gestion des biens des paroisses. Cette position peut se résumer en deux mots: la responsabilité effective de toute l'administration temporelle appartient au curé et à lui seul. Le législatur de 1917 centralise cette tâche entre les mains du curé et exclut, 'par le fait même, une participation effective - nous disons bien effective - des laics. Dans l'optique du Code, le vrai administrateur est le curé. Les laics, si on les admet dans les conseils d'administration, n'y tiennent qu'un rôle de figurants; on ne leur donne pas de responsabilité réelle.

Cette tendance à faire du curé le vrai et unique responsable effectif de l'administration des biens se dégage de différents textes du Codex iuris canonici. En effet, selon le canon $1182 \S 1$, l'administrateur normal des biens de l'église paroissiale est le curé. La gestion de ces biens lui incombe; il en est le maitre absolu, en se conformant bien entendu aux règles fixées par le drait canonique. Sans doute, la loi autorise l'évêque à adjoindre au curé un conseil d'administration; mais il ne lui en fait pas une obligation, comme cela résulte des termes du canon $1183 \S 1$ : Si alii quoque, sive clerici sive laici, in administrationem bonorum alicuius ecclesiae cooptentur... On ne peut pas même dire, d'apres ce texte, que le legislateur souhaite la constitution de tels conseils; il les autorise, si on les juge utiles. C'est tout. L'évêque pourrait donc tout aussi bien laisser les curés gérer, seuls, les fonds de la paroisse. Ainsi, le Code souligne le caractère facultatif de ces conseils. Cependant, dans la plupart des diocèses, les évêques ont prescrit aux curés de s'adjoindre un conseil d'administration pour la gestion des biens matériels des églises. Conformément à une vieille tradition, le Code donne à ce conseil la dénomination de conseil de fabrique (consilium fabricae ecclesiae). Certaines législations particulières ont repris ce terme; d'autres ont adopté une dénomination différente. 
Admettons que l'évêque exige l'institution d'un conseil de fabrique dans les différentes paroisses de son diocèse, comme le droit l'y autorise. Dans ce cas, le Code définit les attributions de ce conseil et dans la définition des attributions transparait, une fois de plus, l'idée que la responsabilité effective revient au cure et non aux membres du conseil. $D$ 'après la règlementation établie par le canon 1183 du Code, le conseil de fabrique, là où il existe, est constitué de la manière suivante: le nombre des membres devant former le conseil est laissé à discrétion de l'évêque; la nomniation et la révocation des membres appartiennent également au chef du diocèse: Huius consilii sodales... nominantur ab Ordinario eiusve delegato et $a b$ eodem possunt ob gravem causam removeri (can. $1183 \S 2$ ). Le Code n'accorde en principe ni au curé, ni encore moins aux paroissiens un droit quelconque de désignation ou de proposition. L'évêque reste donc maître, s'il le désire, de la nomination et de la révocation des conseillers. La présidence du conseil est toujours exercée par le curé: Eoque (= rectore ecclesiae) praeside (can. $1183 \S 1$ ). Enfin, le conseil est strictement limité dans ses pouvoirs et dans les matières qui relévent de sa compétence.

Le conseil est limité dans ses pouvoirs: ainsi, les membres du conseil de fabrique ne jouissent que la voix consultative et non de la voix délibérative. Certes, cette restriction n'est pas formulée de façon explicite dans les canons 1182 à 1186, qui traitent de l'administration des biens des églises; mais elle se trouve formulée au canon $1520 § 3$ au sujet des conseillers qui entourent l'évêque pour la gestion des biens. On peut en conclure que les mêmes principes sont applicables aux conseillers qui sont adjoints au curé, d'autant plus que le canon $1182 \S 1$ détermine les mêmes règles pour toutes les églises, qu'elles soient cathédrales, collégiales, paroissiales. Et c'est bien de cette façon que les législations particulières ont interprété la loi du Code. Cela revient à dire que le curé est tenu de requérir seulement l'avis des fabriciens et non leur consentement. Par conséquent, tout en étant astreint d'entendre son conseil, le curé n'est pas obligé de se rallier aux vues de la majorité; il peut agit contre l'avis de la majorité de son conseil, conformément aux dispositions du canon 105 du Code de 1917. Sa décision sera parfairement valide.

En outre, le conseil de fabrique est strictement limité dans ses attributions; les matières qui relèvent de sa compétence ont été dèterminées avec minutie. Le rôle du conseil se borne à la gestion des biens matériels appartenant à l'église; il lui est interdit de s'immiscer dans le domaine spirituel, qui est exclusivement réservé au curé. Pour éviter tout malentendu, le législateur a pris soin d'énumérer au canon 1184 quelques-uns des domaines où les membres du conseil de fabrique pourraient être tentés de s'ingérer et 
qui ne sont pas de leur compétence. Cec domaines sont les suivants: l'exercice du culte lui-méme; la fixation de l'heure des offices, de leur durée, de leur nombre; la police à l'église; l'usage des cloches; la règlamentation des quêtes, des publications, des annonces; la disposition du mobilier; le choix des objets servant au culte ou à l'ornementation (statues, tableaux, luminaires...); la tenue des registres paroissiaux. L'énumération n'est pas limitative, car le canon 1184, qui donne la liste des matières échappant à la compétence du conseil de fabrique, porte la mention: praesertim. Toutes ces affaires et d'autres, bien qu'elles impliquent souvent un élément matériel, relévent de l'administration spirituelle; elles sont de la compétence exclusive du curé et ne font pas partie des attributlons du conseil de fabrique. En aucune de ces matières, le curé n'est tenu de requérir l'avis de ses conseillers. La même exclusive vaut pour le choix du personnel de l'église: organiste, sacristain, chantre et autres personnes utiles au service du culte. Le canon 1185 du Code en soustrait le choix au conseil de fabrique, bien ce personnel soit rétribué sur les fonds dont le conseil assume la gestion. La désignation, la surveillance et le renvoi de ce personnel sont un droit exclusif du recteur de l'église, en l'occurrence du curé:... a solo ecclesiae rectore... nominantur, pendent, dimittuntur.

Ainsi, la tendance à centrasiler toute l'administration temporelle de l'église paroissiale entre les mains du curé et d'en exclure la participation des laics est manifeste; elle se dégage des différents canons du Codex iuris canonici concernant le conseil de fabrique. Et il faut bien conveir que cette législation n'est pas restée lettre morte. Pour s'en convaincre il suffit de parcourir les statuts synodaux de quelques diocèses dans des pays qui ne ront pas liés par des conventions concordataires ou autres. La lecture des status synodaux postérieurs à 1917 laisse apparaitre qe les dispositione du Code ont été obserées de façon très stricte: l'évêque et le curé sont les responsables effectifs de l'administration des biens. La participation des laics à cette responsabilité est réduite au minimum, bien qu'il s'agisse des biens matériels, done d'un secteur où les laics ont, en règle générale, une expérience et une compétence supérieures à celles des clercs. Nous nous contentons de renvoyer le lecteur, à titre d'information, aux différents statuts synodaux édictés après 1917 en France. D'après ces textes, toute l'administration des biens de la paroisse est entre les mains du cure, qui est de droit président du conseil. La désignation des membres du conseil est faite par l'autorité ecclésiastique. La rôle des conseillers est d'ordre purement consultatif 1.

1 On trouvera quelques extraits de status synodaux francais postérieurs à 1917 dans une etude que nous avons publiée dans les Mélanges 
Le IIe concile du Vatican conçoit les choses d'une façon toute différente. Alors que le Code de 1917 écartait les laics de toute responsabilité effective, Vatican II au contraire les incite à une réelle participation. Le concile s'efforce de donner aux laics une véritable place dans l'Eglise; il les convie à travailler avec la hiérarchie à l'oeuvre de salut telle qu'elle esit appelée à se réaliser dans l'Eglise et par l'Eglise. Cet appel à collaboration n'a de sens que si les laics se voient confier des responsabilités affectives. Or s'il est un domaine où l'activité des laics est appelée à s'exercer de façon afficace, c'est sans ausun doute celui de la gestion des biens matériels.

Certes, contrairement au Code de 1917, Vatican II ne prend pas de dispositions l'egislatives concrètes à ce sujet; il se contente de donner des orientations. Mais ces orientations sont tellement nettes que le futur législateur sera obligé de les traduire dans les textes juridiques en attribuant aux laics une responsabilité véritable, sinon il ne serait pas fidéle à l'esprit du concile. Les textes dans lesquels Vatican II donne les orientations relatives à la responsabilité des laics sont de deux sortes: les uns mentionnet d'une facon générale cet appel à la collaboration 2; les autres font ex plicitement allusion à la gestion des biens. Nous nous contentons de citer deux textes de la seconde catégorie, c'est-à-dire des textes qui mentionnent, de façon explicite, l'administration des biens et émettent le souhait que les laics y prennont des responsabilités. Ainsi nous lisons dans le décret sur le ministère et ia vie des prêtres, Presbyterorum ordinis, au $n^{\circ} 17$ : „Quant aux biens ecclésiastiques proprement dits, les prêtres les administreront à leur nature et selon les lois ecclésiastiques, autant que possible avec l'aide de laics compétents" ${ }^{3}$. Le décret sur l'activité missionnaire de l'Eglise, Ad Gentes, est encore plus précis; il demande sans ambages que la gestion des biens soit confiée à des laics, au $n^{\circ} 41$, 3e alinéa: „Dans les territoires des missions, les laics soit étrangers, soit autochtones, doivent... avoir la gestion des affaires temporelles..." 4. Il y a la des éléments suffisants pour inciter l'Eglise postconciliaire à réviser sa conception de la responsabilité des laics quant à la gestion des biens temporels des paroisses.

Il ne fait pas de doute que la manière de voir qui apparaissait dans le Code de 1917 est dépassée; une orientation différente est

Bidagor: Ius populi Dei. Miscellanea in honorem Raymundi Bidagor, t. 2, Roma 1972 p. $212-215$.

2 Voir, entre autres, la constitution Lumen Gentium, $n^{\circ} 37 \S 3$ et 4 , et le décret Apostolicam actuositatem, $\mathrm{n}^{\circ} 10$.

${ }^{3}$ Nos citations sont faites d'après l'édition, en un volume: Concile oecuménique Vatican II, textes, français et latin, Paris Centurion, 1967. p. 434 .

4 Edit. cit., p. 600. 
donée par Vatican II. D'après la nouvelle conception, la responsabilité de la gestion des biens doit essentiellement revenir aux laics; ou, pour le moins, une responsabilité réelle et une possibilité d'action affective doivent leur être atribuées. Le monopole de la responsabilité effective que le Code octroyait au curé n'est plus défendable.

Telles sont les deux orientations, bien différentes, devant lesquelles nous mettent la législation de 1917 et les documents de Vatican II: d'un côté, pratiquement un refus de confier aux laics une responsabilité effective dans la gestion des biens de la paroisse; de l'autre, une invitation pressante adressée aux laics de prendre une responsabilité affective dans ce même domaine. Il s'agit là d'un constat: nous nous trouvons en prèsence de deux conceptions, qui non seulemet divergent, mais marquent une opposition très nette. Il nous intéresse de connaître les motifs qui sont à l'origine de cette double orientation, diamétralement opposée.

\section{Les motifs du changement d'orientation}

Il importe de rechercher les raisons pour lesquelles en 1917 l'Eglise se soit refusée de confier aux laics une participation réelle dans la gestion des biens et au IIe concile du Vatican au contraire elle ait sollicité, avec insistance, les laics à prendre les responsabilités qu'elle leur avait déniées en 1917.

On pourrait être tenté de juger sévérement nos devanciers, qui ont ainsi minimisé le rôle des laics en les écartant de l'administration effective des biens matériels. Ce faisant. nous serions certainement injustes à leur égard; car pour porter un jugement équitable, il faut toujours se rappeler qu'une législation reflète l'esprit et la mentalité d'une époque. Or la législation de 1917 marque l'aboutissement du droit d'une Eglise qui a été longtemps liée au pouvoir civil. L'Eglise catholique a fait l'expérience, pendant les derniers siècles, d'une immixtion trop grande du pouvoir laic dans le domaine spirituel. On en trouve l'illustration tous les ouvrages d'histoire tant soit peu complets qui traitent des relations de l'Eglise catholique et du pouvoir étatique aux XVIIIe et XIXe siècles ${ }^{5}$. Après le gallicanisme, il suffit d'évoquer, entre autres, le joséphisme, le fébronianisme, la lutte menée en Allemagne à l'époque du Kulturkampf pour voir surgir, devant l'Eglise catholique, des spectres qui menaçaient sa liberté d'action. Aussi l'Eglise s'efforça-t-elle de sa dégager des entraves que lui

5 Nous nous contentons de renvoyer à deux ouvrages généraux: H. E. F e in e, Kirchliche Rechtsgeschichte, 4. Aufl., Köln-Graz, 1964, pp. 573-599, 628-650, 669-688; Handbuch der Kirchengeschichte, hrsg. von H. Jedi n, t. VI, Die Kirche der Gegenwart, 1. Teil Freiburg i.B., 1971 , pp. $348-414,696-760 ; 2$. Teil, 1973, pp. 28-194, 501-549. 
avaient imposées certains gouvernements et qui mettaient de sérieux freins à son activité dans maints domaines. Au cours de la seconde moitié du XIXe siècle et au début du XXe, plusieurs facteurs et circonstances avaient contribué à libérer l'Eglise de l'emprise du pouvoir séculier dans divers pays et, malgré des inconvénients immédiats d'ordre matériel essentiellement, lui avaient assuré à longue échéance une grande liberté d'action dans le domaine interne et spirituel ${ }^{6}$. C'est à ce moment que les canonistes élaborent le concept de la societas perfecta, appliqué à l'Eglise ? Aussi Hans Erich Feine note-t-il, à la suite de Ulrich Stutz, qu'à la fin du XIXe siècle le droit canonique se spiritualise de plus en plus; il parle d'une fortschreitende Spiritualisierung des Kirchenrechts après $1870^{8}$. Nous parlerions plus volontiers d'un désengagement temporel de la part de l'Eglise, bien que l'expression soit récente et puisse paraître anachronique pour l'époque.

Dans ce contexte, on comprend fort bien la mentalité des canonistes qui ont été chargés de rédiger le nouveau Code au début, $\mathrm{du} \mathrm{XX}$ siècle. Ces canonistes avaient été les témoins d'une Eglise qui était hantée par l'immixtion de l'autorité civile dans ses affaires et qui avait réussi à s'en affranchir au prix de luttes et de grands sacrifices. Ils auront le souci d'éviter, à tout prix, que l'Eglise retombe sous la tutelle laique; ils prendront les mesures en conséquence. C'est pourquoi les dispositions que l'on trouve dans la législation de 1917 pour écarter les laics des responsabilités effectives s'expliquent, en trés grande partie, de cette manière.

On a voulu, en 1917, édifier une société ecclésiastique libre de toute emprise laique. Ce fut réaction fort compréhensible dans le contexte de l'époque; mais comme toute réaction elle a péché par

- Parmi ces factures ou circonstances historiques, on peut signaler la perte des Etats de l'Eglise en 1870, la séparation de l'Eglise et de l'Etat intervenue dans certains pays, les accords ou concordats fondés sur la réciprocité conclus avec d'autres pays. Certes, il n'y a pas de ligne continue dans l'évolution des relations de l'Eglise et des Etats; elle varie d'un pays à l'autre. On peut simplement dégager une ligne très génèrale, qui va dans le sens d'une autonomie des deux sociétés, la civile et la religieuse.

7 Cf. Alberto de la Her a et Charles M unier, Le droit public ecclésiastique d travers ses définitions, dans Revue de droit canonique, t. 14,1964, p. $32-63$; surtout p. 53 ss. Il convient de noter que les auteurs de cette étude n'ont pas fait le lien entre l'appartition du concept de "société parfaite" dans le droit public ecclésiastique et la perte des Etats de l'Eglise; or il semble bien que c'est la dispartion du pouvoir temporel qui est à l'origine de l'inistance avec laquelle les canonistes appliquent, surtout après 1870 , ̀̀ l'Eglise la notion de la societas perfecta. Ce fut un moyen pour l'Eglise de compenser quelque peu la perte de ses Etats, grace auxquels l'Eglise catholique avait eu une place parmi les autres Etats à souveraineté territoriale.

8 Fein e, op. cit., 4; voir aussi p. 658 ss. 
excès. Sous prétexte de rendre l'Eglise libre, on a crée pour ainsi dire le vide autour d'elle: la rsponsabilité ds laics est devenue pratiquement inexistante dans l'organisation de l'Eglise. Mais qui oserait jeté la pierre è nos devanciers? Leur attitude a été dicté par un geste d'auto-défense de la liberté, dont l'Eglise ávait été trop longtemps privée dans divers pays. En outre, il convient de signaler un autre élément fort important, qu'on oublie peut-être trop souvent: en écartant les laics des responsabilités dans la gestion des biens, le Code de 1917 était dans l'authentique ligne de la tradition de l'Eglise primitive.

Contrairement à ce qu'on pourrait penser, les laics n'ont jamais tenu un rôle prépondérant dans la gestion des biens matériels. Nous parlons du droit commun de l'Eglise et non du droit particulier; car il serait facile de citer des exemples pris dans certains pays ou certaines régions, dans lesquels on faisait la part belle aux laics surtout à partir du XVIII e siècle. Mais, en vertu de la législation canonique générale, la responsabilité dévolue aux laics dans l'administration des biens temporels n'a jamais été très importante; pour s'en convaincre il suffit de ce livrer à une rapide enquête historique.

Durant le premier millénaire, le gestion des biens des églises relevait de la compétence quasi exclusive de l'autorité ecclésiastique. Ce fut particulièrement vrai aux origines de l'Eglise. Rudolph Sohm (1841-1917) a fort bien expliqué la raison d'être de cette pratique pour le Ier siècle. Selon le grand canoniste protestant, l'exclusion des laics et le monopole de l'autorité ecclesiastique en ce qui concerne la gestion des biens temporels de la communauté ecclésiale était fondée sur des motivations d'odre ecclesiologique. On sait que Somh reste l'éternel gêneur pour les canonistes 9. Mais il faut bien reconnaitre que si dans l'ensemble on ne peut pas suivre Sohm dans l'interprétation qu'il donne de l'évolution du droit de l'Eglise, on trouve cependant des éléments acceptables dans beaucoup de ses affirmations $\mathbf{1 0}^{0}$.

Nous nous permettons de rappeler, très brièvement, la manière dont Sohm a expliqué l'exclusion des laics: il l'a fait dans le premier tome son ouvrage: Kirchenrecht 11. A l'origine, la célébration de l'eucharistie était le point culminant dans la vie dela com-

9 Voir à ce sujet, entre autres, Y. Cong a r, R. Sohm nous interroge encore, dans Revue des sciences philosophiques et théologiques, t. 57. 1973, p. $263-294$. En annexe de cet article figure une bibliographie d'études relatives à la pensée de Sohm.

${ }^{10}$ Bien des auteurs qui à l'heure actuelle critiquent certaines institutions de l'Eglise, ne font que reprendre, sans le savoir bien souvent, des idées avancées par Sohm, il y a bientôt un siècle.

${ }^{11}$ Kirchenrecht, t. I, Die geschichtlichen Grundlagen, Leipzig 1892 (réimpression Munich, 1923), p. 73 ss. 
munauté chrétienne. C'est autour de la célébration de l'eucharistie que se forma le premier noyau d'une organisation ecclésiale. Les chrétiens apportaient des biens, sous la forme d'aumônes, à l'occasion de la célébration eucharistique. Ces biens, dons et aumones des fidèles, étaient offerts à Dieu; ils n'étaient donc pas les biens de la communauté, mais les biens du Seigneur. Aussi la communauté n'était-elle pas habilitée à les gérer, contrairement à ce qui se serait passé si la communauté chrétienne avait eu le caractère d'une association du type des associations que l'on rencontrait dans la société de l'époque. Si la communauté chrétienne avait été une association semblable aux autres, elle aurait été compétente pour gérer ces biens et elle aurait pu déléguer ses pouvoirs au président ou à quelque comité. Or les aumônes données par les chrétiens à l'occasion de la réunion eucharistique ne deviennent pas les biens de la communauté; ce sot les biens de Dieu. Ils ne peuvent donc être administrés qu'au nom de Dieu. Celui qui représente Dieu au sein de la communauté est seul compétent et responsable de la gestion de ces bins. C'est pourquai dans la communauté primitive l'administration des dons et aumônes était assurée par les apôtres et par les seipt diacres, qui apparaisseaient comme dotés par Dieu de qualités spéciales pour cet office. A la mort des apôtres, ceux qui les remplaceront à la tête de la communauteé rempliront cette fonction.

Cette idée que les biens, constitués par les dons et aumônes, étaient les biens de la communauté et que la communauté pouviaient les gérer et en disposer est une idée inconnue dans l'Eglise primitive. Comme Sohm le fait bien remarquer, l'administration des biens n'était pas réglée selon le principe communautaire des biens d'une association, mais selon le principe autoritaire: Auch für die Vermögungsverwaltung gilt nicht Gemeindeprincip im modernen Sinne des Wortes, sondern Autoritätsprincip: Verwaltung nicht kraft Gemeindeauftrags, sondern kraft des Auftrags, welcher von oben her, von Gott durch das Charisma gegeben word ist 12. Cela signifie que l'administration n'était pas faite en vertu du mandat reçu de la communauté, mais en vertu du mandat reçu d'en haut, du mandat reçu de Dieu.

Telle est rapidement esquissée la manière dont $\mathrm{Sohm}$ voit la gestion des biens l'Eglise primitive. D'après ce qu'il dit, on comprend que les laics n'aient eu aucune part à cette gestion. La responsabilité en incombait uniquement à l'autorité, plus exactement à celui qui représentait Dieu au sein de la communautè, à celui à qui Dieu avait communiqué son autorité.

Il semble que l'interprétation donnée par Sohm contienne une bonne part de vérité. En tout cas, les faits, disons l'histoire, con-

12 S o h m, op. cit., p. 77--78. 
firment son interprétation. Effectivement, comme nous l'avons déjà indiqué, durant le premier millénaire, la responsabilitè de gerer les biens temporels de l'Eglise relèvent de la compétence exclusive de l'autorité cclésiastique. De toute façon, il n'est guère question de responsabilité partagée avec les laics; les quelques sondages que nous avons faits dans les textes des conciles et autres sources des dix premiers siècles pour trouver des indications sur une participation effective des laics à la gestion des biens des églises, se son avérés sans résultat ${ }^{13}$. Cet état de choses se poursuit pratiquement jusqu'au XIIe siècle ${ }^{14}$. Ce n'est qu'à partir de cette époque que nous trouvons des textes mentionnant la participation des laics à l'administration des biens des églises. Rares au XIIe et au XIIIe siècle, les textes se font plus nombreux dans la suite. L'Eglise finit par confier à des laics une part de responsabilité en ce domaine, mais elle n'entend pas leur réserver une place de choix; en vertu du droit commun, le curé reste toujours le maitre de cette administration ${ }^{15}$. C'est cette législation ou cet état de fait que continue à admettre de façon implicite le concile de Trente ${ }^{16}$ et qui se perpétuera pratiquement jusqu'à la veille

13 Sur la situation durant le premier millénaire, oú l'ádministration des biens a été le monopole de l'autorité ecclésiastique, voir $\mathrm{L}$. $\mathrm{T}$ h omassin, Ancienne et nouvelle discipline de l'Eglise, édit, André, t. VI, Bar-le-Duc, 1866, p. 509 ss. Une enquête faite dans les excellents travaux que Mgr Lesne a consacrés à la propriété ecclésiastique jusqu'au XIIe siècle n'a fourni qu'un résultat négatif; nous n'y avons relevé aucune indication concluante sur la participation effective des laires à la gestion des bien des églises: Emile Les ne, Histoire de la propriété ecclésiastique en France, it. I-VI, Lille, 19101943, dans Mémoires et travaux publiés par les professeurs des Facultés cath. de Lille fasc. 6, 19, 30, 34, 44, 46, 50, 53 .

14 Nous trouvons encore au XIIe siècle des textes très significatifs d'Alexandre III (1159-1181), qui confirment cet état d'esprit. Voir, par exemple, un texte d'Alexandre III, reproduit dans les Décrétales de Grégoire IX, 1, 14, 2: i] est question d'un évêque qui a concédé des églises à des enfants, qui n'avaient pas même l'âge de 10 ans; en attendant que les intéressés aient atteint l'âge requis pour être en mensure d'administrer eux-mêmes lesdites églises, l'évêque en a confié la gestion à des laics et non à des clercs. Ce que le pape blâme essentiellement, ce n'est pas le fait d'avoir donné des églises à des enfants, mais d'en avoir confié provisoirement l'administration à des laics; les laics devaient sans doute administrer, avant tout, les revenus de ces églises, car on ne voit pas dans quelle mesure des laics auraient pu gérer les affaires spirituelles.

${ }_{15} \mathrm{Au}$ sujet de l'évolution historique de la participation des laics à l'administration des biens des èglises à partir du XIIe siècle, on trouve de bons éléments dans l'ouvrage dèjà cité de L. Thom as $\mathrm{s}$ in, Ancienne et nouvelle discipline de l'Eglise, édit. André, t. VII, Bar -1 e - D u c, 1867 , p. $65-69$.

${ }_{16}$ Le concile de Trente n'apporte pas le précision sur la part réservée aux laics; il se contente d'exiger des administrateurs laiques et ecclésiastiques une reddition des comptes à l'évêque (sess. 22, de ref., 
du Code de 1917 dans le droit commun: pour s'en convaincre, il suffit d'ouvrir l'un des grands traités de droit canonique qui faisaient autorité avant 191717 . Le Codex iuris canonici renforcera encore, si besoin était, le rôle prépondérant du curé et minimisera la part des laics. Cette façon de voir répondait aux préocupations de l'Eglise à la fin du XIXe siècle et au début du XXe, comme nous l'avons déjà indiqué.

En somme, l'exclusion des laics de toute responsabilité effective dans la gestion des biens temporels, au moins dans le droit commun, correspond à une tradition continue qui va des origines de l'Eglise jusqu'au XXe siècle. Vue de l'extérieur, l'institution est restée la même, mais - et cela paraît important - à regarder de plus près on constate un changement dans les motifs qui tendent à exclure les laics et à confier toute la responsabilité à l'autorité ecclésiale. Cette évolution est fort curieuse et mérite réflexion. A l'origine, à l'époque de l'Eglise naissante, les motivations pour lesquelles on exclut les laics sont des motivations d'ordre ecclésiologique: on écarte les laics, non pas parce qu'on a peur de leur immixtion dans les affaires de l'Eglise, mais parce que l'Eglise n'est pas une association semblable aux associations que l'on rencontre dans la société civile. Elle est une communauté sui generis. Seul le représentant de Dieu est compétent pour gérer les biens offerts par les fidèles, car ces biens sont les biens de Dieu et non ceux de la communauté. Le représentant de Dieu est celui qui préside l'eucharistie.

Dans la suite, la responsabilité des biens des églises est restée aux mains des représentants de Dieu, à savoir l'évêque, le curé, au moins là où l'Eglise était libre d'agir à sa guise. Mais les motivations n'ont plus été tout à fait les mêmes. Un glissement s'est apéré peu à peu; certes, cela s'est fait de façon inconsciente. On continuera d'écarter les laics, sans se rendre compte que lés motifs pour lesquels on les écartait, n'étaient plus ceux qui avaient joué à l'origine. Ce glissement ou ce changement de motivation est particulièrement sensible à fin du XIXe siècle et au début du $\mathrm{XXe}$. On se conforme de façon très stricte, là où les circonstances le parmettent, à la manière d'agir qui avait été celle de l'Eglise primitive. Mais les motifs sont d'un autre ordre. En tout cas, ils ne sont plus d'ordre ecclésiologique; ces motifs, on les a oubliés. On

c. 9). C'est dire que le concile de Trente admet la participation des laics à la gestion des biens, là où elle existe en vertu du droit particulier; il reconnaît le fait, sans donner de règlementation précise.

17 Au sujet du droit commun valable à la veille de la promulgation du Codex iuris canonici, voir Fr. X. W e r nz, Ius Decretalium, t. III/I, Ius administrationis ecclesiae catholicae, 2a edit., Romae, 1908, $\mathrm{n}^{\circ} 190-$ 192 ; J. B. S ä g m ü $11 \mathrm{er}$, Lehrbuch des katholischen Kirchenrechts, 3 Aufl., t. II, Freiburg i.B., 1914, p. 472. 
exclut les laics de la gestion des biens, comme de toute participation, parce, qu'ils sont devenus gênants. L'autorité ecclésiastique veut être maître chez elle: aux motifs d'ordre ecclésiologique ont fait place des motifs d'auto-défense; d'autres diraient, des motifs d'ordre clérical. L'auto-défense ou le désir d'indépendance de l'Eglise motivait au début du XXe siècle l'exclusion des laics, alors qu'à l'origine l'ecclésiologie commandait cette exclusion.

Ainsi, considérée du point de vue institutionnel, la conception n'a pas changé depuis les origines jusqu'au IIe concile du Vatican, au moins dans ce qu'on appelle le droit commun: la responsabilité de la gestion des biens est restée le monopole de l'autorité l'Eglise. Mais les motivations ont évolué: tout au début, nous trouvons des motivations d'ordre ecclésiologique; dans la suite, des motivations d'auto-défense. Nous avons là le cas typique d'une institution dont la structure est restée identíque à elle-même, mais qui a été vidée de son contenu primitif; l'historien des institutions connaît maints exemples de ce genere ${ }^{18}$.

Le IIe concile du Vatican a changé la conception admise jusquelà, en ce qui concerne la responsabilité des laics; le changement de conception entraînera nvcessairement une modification de la structure institutionnelle. Au lieu de refuser aux laics les responsabilités, le concile les invite au contraire à en prendre le maximum dans les domaines qui ne relévent pas directement du pouvoir d'ordre; la gestion des biens matériels appartient, en premier lieu, à ces domaines. Les raisons qui expliquent la nouvelle orientation sont, à notre avis, de deux sortes. La première raison est d'ordre historique: à l'heure actuelle, au moins dans beaucoup de pays, l'Eglise a acquis une liberté d'action telle qu'elle n'est plus à la merci du pouvoir séculier. Elle n'a plus rien à craindre d'une immixtion des laics; bien au contraire, elle souffre de leur absence. La seconde raison est d'ordre ecclésiologique. Alors qu'à l'origine on écartait les laics pour des motifs parfaitement valables du point de vue ecclésiologique, Vatican II invite les laics aux responsabilités pour des motifs non moins valables du point de vue ecclésiologique. Le concile a insisté sur l'idée du sacerdoce universel; de la sorte, il a remis lec laics à leur vraie place dans la communauté ecclésiale. A ce titre, la participation effective aux responsabilités est parfaitement justifiée; elle correspond à l'ecclésiologie développée par Vatican II. Il suffit de lire, à ce sujet, ce que dit le concile dans le décret sur l'apostolat des laics, Apostolicam actuositatem, au $\mathrm{n}^{\circ}$ 10: „Participant à la fonction du Christ Prêtre, Prophète et Roi, les laics ont leur part

18 On relira avec profit à ce sujet l'ouvrage de Gabriel Le Bras, Prolégomènes, Paris 1955, dans Histoire du droit et des institutionis de l'Eglise en Occident, t. 1; voir surtout pp. 38-39 et 188-189. 
active dans la vie et l'action de 'Eglise..." 19. On ne peut être plus clair.

Nous sommes arrivés au terme de la rapide enquête que nous nous étions proposé de faire au sujet d'une institution qui ne tiendra qu'une place de second plan dans la grande réforme des structures de 1'Eglise, engagée par Vatican II. A cette occasion, nous avons constaté, une fois de plus, combien les institutions reflètent la mentalité de l'esprit d'une époque: ceux qui, avant nous, ont été chargés, au cours des siécles, des réformes dans l'Eglise ont scruté les signes de leur temps. Et il faut bien convenir que l'histoire des institutions nous donne une leçon d'humilité. En effet, en jetant ce regard sur le passé, nous avons constaté que pour adapter les institutions aux exigences du temps, on trouve toujours une justification, même théologique ou ecclésiologique. Pendant près de vingt siècles, on a écarté les laics des responsabilités dans le domaine des biens matériels, en s'appuyant tout d'abord sur le fait que les biens de la communauté chrétienne étaient les biens de Dieu, puis en mettant en avant la liberté et l'indépendance de l'Eglise. Dans la seconde moitié du XXe siècle, les circonstances incitent l'Eglise à faire appel à la participation des laics, qu'elle leur avait jusque-là refusée dans la domaine des biens temporels. La nouvelle orientation trouve, elle aussi, un parfait appui dans l'ecclésiologie; il s'agit de l'ecclésiologie développée par Vatican II.

C'est dire combien la part de l'élément humain est important dans beaucoup d'institutions de l'Eglise. L'essentiel est de s'en rendre compte. Ce constat facilite la tâche de ceux qui ont la lourde responsabilité de scruter les signes des temps, comme l'ont fait nos devanciers, et de renouveler les institutions, en ce qu'elles ont d'humain, pour les mettre en harmonie avec l'évolution de la société. Il permet aussi de porter un jugement de valeur plus équitable sur le passé.

\footnotetext{
${ }^{19}$ Les passages dans lesquels le concile mentionne le sacerdoce commun des chrétiens, sont nombreux: Lumen Gentium, 10, 11, 26, 31; Ad Gentes, 15; Apostolicam actuositatem, 3; De sacra liturgia, 14 etc...

5 - Prawo kanoniczne
} 